\title{
Characteristics of radio spikes as solar microflares at short decimetric wavelength
}

\author{
Rui Xiang Xie ${ }^{1}$, Min Wang ${ }^{1}$, Qi Jun $\mathrm{Fu}^{2}$, and Wei $\mathrm{Li}^{2}$ \\ 1 Yunnan Observatory, National Astronomical Observatories, CAS, Kunming 650011, PR China \\ 2 National Astronomical Observatories, CAS, Beijing 100012, PR China
}

Received 8 October 1999 / Accepted 31 July 2002

\begin{abstract}
A rare type of radio spike occurred on January 5, 1994 at short decimetric wavelength (1.42 GHz), and was observed with the Yunnan Observatory high time resolution $(1 \mathrm{~ms})$ radio telescope. The radio and optical characteristics of the spikes as radio microflares are presented. This event contains 53 radio millisecond spikes, and shows an intermittently periodic pulse train superimposed on the radio continuum background. Two newly emerging small sunspot groups with complex polarities were observed. The magnetic field strength of the source and the irradiated spike energies are estimated by using the radio observations. During a single spike, $\leq 10^{23}$ ergs may have been transferred to nonthermal electrons, and thus radio spikes may reflect microflare class activity. We discuss the generation mechanism of the radio microflares qualitatively. Our results favour the explosive coalescence model of the multiple magnetic flux loops, leading simultaneously to acceleration of electrons and heating.
\end{abstract}

Key words. Sun: flares - Sun: radio radiation - Sun: magnetic fields - Sun: sunspots

\section{Introduction}

Impulsive variations of the radio emission in the corona or transition region, that could be defined as low-energy flares, have long been observed in both quiet-sun regions and active regions. High-resolution observations taken by, e.g., Yohkoh and $\mathrm{SOHO}$ during the solar minimum years have revealed small-scale transient phenomena occurring everywhere on the solar surface (Ulmschneider et al. 1991). In this paper we will focus on the characteristics of radio millisecond spikes. One flare may consist of several thousand microflares (Benz 1985), but not all flare events have observable microflares. Microflares may then require special ambient conditions and acceleration mechanisms. The instantaneous energy release during microflares may generate a variety of radio fast fine structures (FFS), such as millisecond spikes, pulsations (Benz 1985; Aurass et al. 1987), type III bursts from metre to microwave wavelengths (Stähli \& Benz 1987) and transient microwave brightenings (TMBs) (Gopalswamy et al. 1994), etc. During subflares an energy of $\sim 10^{29} \mathrm{ergs}$ is released while $\sim 10^{32}$ ergs is released during the largest flares. On the other hand, large X-ray microflares may dissipate about $10^{24}-10^{26}$ ergs (Lin et al. 1984). A single radio spike may contain $10^{16}-10^{18}$ ergs (Aschwanden \& Güdel 1992), corresponding to $10^{23}-10^{24}$ ergs released at the Sun, thus suggesting that radio FFSs are sensitive indicators of solar

Send offprint requests to: Rui Xiang Xie, e-mail: wmynao@163.net activities. It has also been suggested that particle acceleration occurs at sites where the ambient electronic density corresponds to plasma frequency in the decimetric range (Bastian et al. 1998). The cause of particle acceleration may be due to the changes of magnetic field topology during the impulsive phase (Trottet 1986) and/or loop-loop interactions (Hanaoka 1997). Solar activities have various forms but to some degree all are linked with the emergence of strong magnetic fields in the solar atmosphere which is marked by change in sunspots spatial distribution. The magnetic energy is stored in such active regions and triggered under some favorable circumstances. The explosive release of the pent-up energy will lead to a variety of emissions (Wild et al. 1963). The primary energy release often appears as microflares or elementary flares, i.e. in the form of radio FFSs. Spike sources are close to or in the regions of energy release, therefore, the observations of spikes may be probes of microflares (Benz 1985; Aschwanden et al. 1990). Observational properties of FFSs include short-duration pulse series, double- or triple-peak structures, and have been reviewed (see for example, Fleishman \& Melnikov 1998). FFSs reported in this paper have similar properties although with unusual long (17 s and $34 \mathrm{~s}$ ) periods observed through the flare development.

This paper is not intended to be a study of the emission mechanism of the radio spikes, but rather bring new insights into the unusual properties of the event detected with high time resolution. In Sect. 2 the radio and optical observations are 
presented. In Sect. 3 the results are described. In Sect. 4 the spikes as microflares and its emission process are discussed qualitatively. Finally, a summary and conclusions are given in Sect. 5.

\section{Observations}

\subsection{Radio observations}

A series of radio spikes was observed during 0645-0720 UT on January 5, 1994 by the Yunnan Observatory high time resolution (1 ms) solar radio telescope (Xie et al. 1989). The spikes occurred during a small $4 \mathrm{~S} / \mathrm{F}$ radio burst at $1.42 \mathrm{GHz}$. The active region AR 7646 was located at S14W19. This event was associated with an $\mathrm{H} \alpha$ flare of importance $1 \mathrm{~N}$, an M1.0 X-ray burst and small radio bursts at centimeter to short decimeter wavelengths (see S.G.D. 1994, 594 - Part I). The radio spikes occurred throughout the radio burst at $1.42 \mathrm{GHz}$, with 53 FFSs superimposed on the continuum background. The duration of the spikes range from 15 to $50 \mathrm{~ms}$, with an average of $33.1 \mathrm{~ms}$, and their intensities range from 150 to 200 s.f.u. (1 s.f.u. $=10^{-22} \mathrm{Wm}^{-2} \mathrm{~Hz}^{-1}$ ). Figure 1 shows the time profiles of the radio burst at $1.42 \mathrm{GHz}$ and some samples of typical FFSs. The fluctuating structures consist of some spike-like emissions whose durations are in the range 1 to $3 \mathrm{~s}$ (see the enlarged part in Fig. 1).

Simultaneous observations were also performed at Beijing Observatory, but millisecond spikes were not detected using the high time resolution $(1 \mathrm{~ms})$ radio telescope at $2.84 \mathrm{GHz}$. Only a pair of microwave type III bursts with positive and negative frequency drift was observed (Fu et al. 1997) using the 1.0-2.0 GHz radio-spectrograph (Ji et al. 1997). This pair of type III bursts is superimposed on a small burst, and shows a separatrix frequency of about $1.3 \mathrm{GHz}$ (see Fig. 2).

\subsection{Optical observations}

The $\mathrm{H} \alpha$ flare started at 0648 UT on Jan. 5, 1994, reached its maximum at $0650 \mathrm{UT}$ and ended at 0736 UT (S.G.D. 1994, 594 - Part I). The sunspot groups in the AR 7646 are of moderate size with double polarities. The preceding and following spots are linked by some smaller spots, corresponding to a magnetic field structure of type $\beta \gamma$. We note that newly emerged small spots (S1, S2 and S3 etc. and N4, S4 and S5, etc. in Fig. 3) are located south and northeast of the preceding spots $\left(S 09^{\circ}, \mathrm{L} 89^{\circ} 2\right)$ on Jan. 5 , respectively. The sunspots marked by $\mathrm{S} 1, \mathrm{~S} 2$ and S3 are connected with the major sunspots marked by N1, N2 and N3 in the umbra (Fig. 3). Using a magnetogram (provided by Huairou Solar Station of Beijing Astronomical Observatory) corresponding to the sunspots drawn in Fig. 3 we can see that the opposite polarities marked by N1 - N4 and S1 - S4 exist in the same penumbra and this probably leads to an increase of magnetic field gradient. Moreover, there was a thick and short filament in the east-west direction (Fig. 4, from Yunnan Observatory), which suddenly disappeared just before the maximum of the flare on Jan. 5. These phenomena may have been associated with the generation of radio microflares.

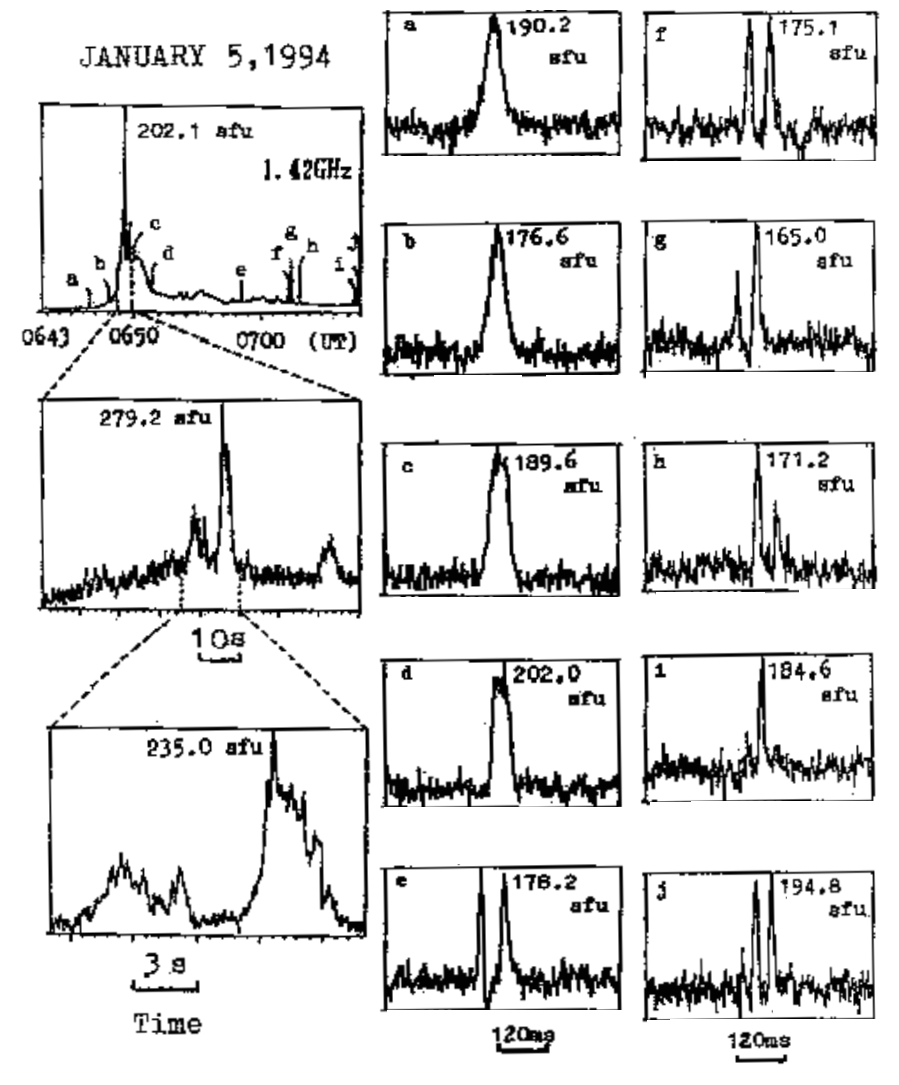

Fig. 1. The time profile of radio burst with the time constant of $1 \mathrm{~s}$ and the magnified profiles corresponding to the intervals marked by dashed lines (left column); The fast fine structures with the time constant of $1 \mathrm{~ms}$ corresponding to $\mathrm{a}, \mathrm{b},-\mathrm{j}$ of the left column, respectively (middle and right).

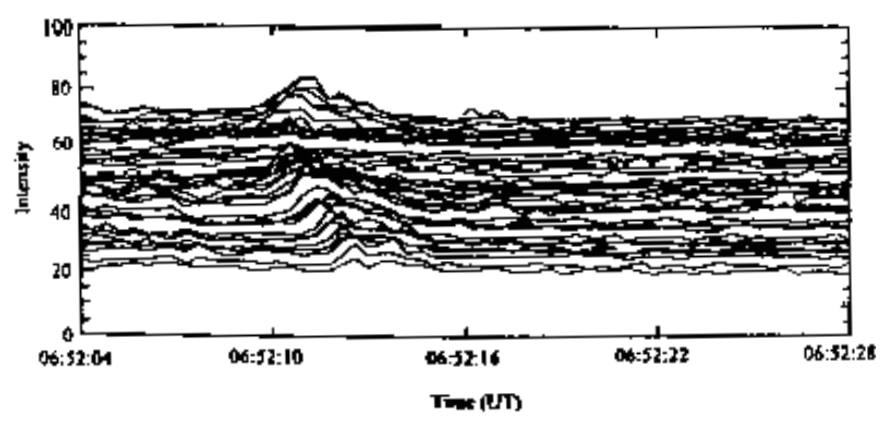

Fig. 2. The time-evolving radio spectra of a pair of type III bursts superposed on the peak of the burst on January 5, 1994. The intensity scales are uniform and the average intensity is about 10 s.f.u.; The frequency range of the plots from upper to lower represent $1-2 \mathrm{GHz}$ (From Fu et al. 1997).

\section{Results}

\subsection{Estimation of irradiated energy of the spikes}

The energy released from each spike can be estimated by assuming that the emission is due to a coherent emission process. Adopting typically observed frequency bandwidths of $10 \mathrm{MHz}$, we obtain the radio energy content of each spikes by integrating its total flux density time profile. We found that the radio energy radiated during a single spike is of the order of 


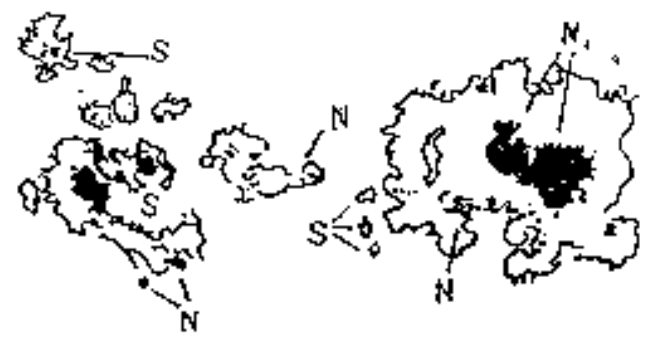

$5^{-}$Januaty'4,1994 0I:25 LT

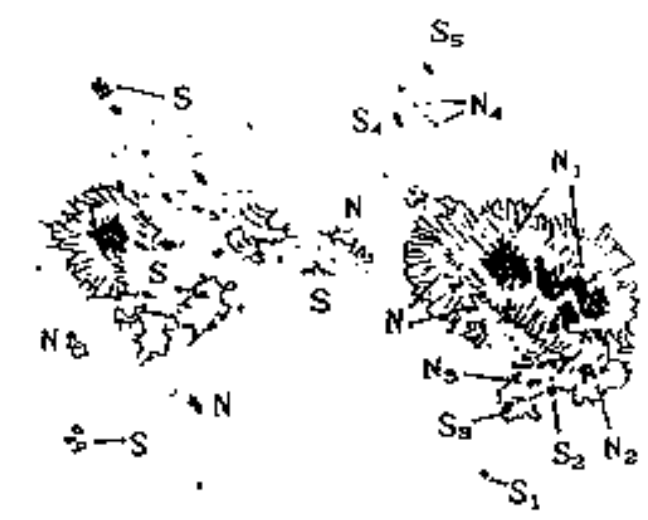

Januaty 5,1994 03: 11 ㄴ?

Wave Length : $25324 \mathrm{~A}$

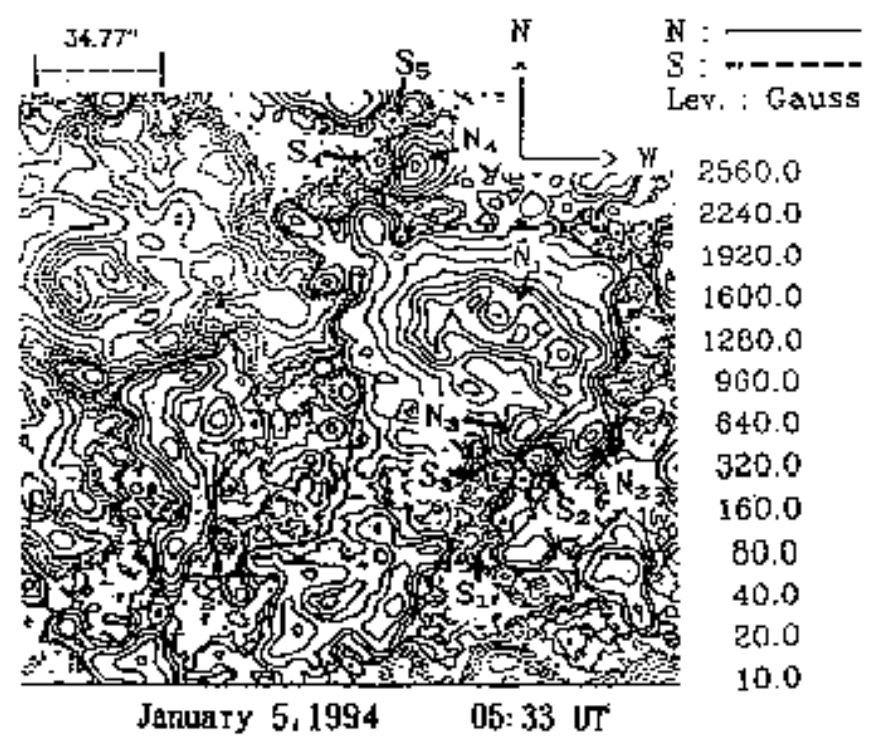

Fig. 3. An overview for comparison of the sunspots and the corresponding magnetogram in AR 7646. The hand-drawing sunspot groups of 94/01/04 and 94/01/05 (Yunnan Obs.), N1 - N4 and S1 - S5 represent the newly emerging sunspots, respectively (top and middle panels). The magnetogram (Huairou Solar Station, Beijing Astro. Obs.) corresponding to sunspots (N1 - N4 and S1 - S5) represents the different polarities, respectively (bottom panel).

$(0.3-3.3) \times 10^{17}$ ergs. Because spike production involves collective processes, it is difficult to relate the above radio energy content values to the energy actually transferred to the non-thermal electrons responsible for FFSs. However, the latter
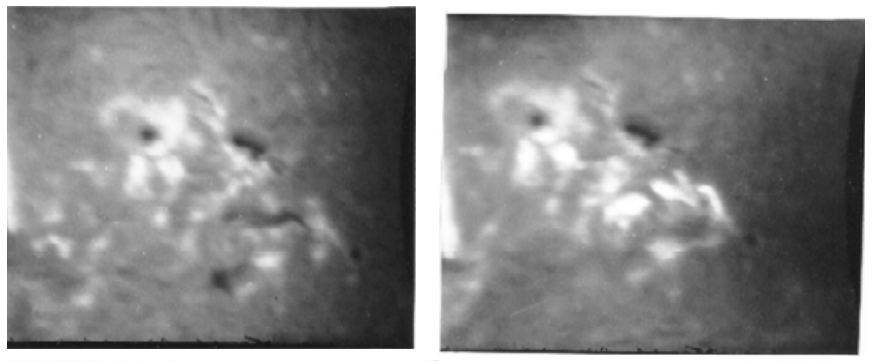

Fig. 4. $\mathrm{H} \alpha$ image from Yunnan Observatory, showing the filament between the two groups of sunspots. It appeared before 064120 UT (left frame), and disappeared at 064904 UT (right frame).

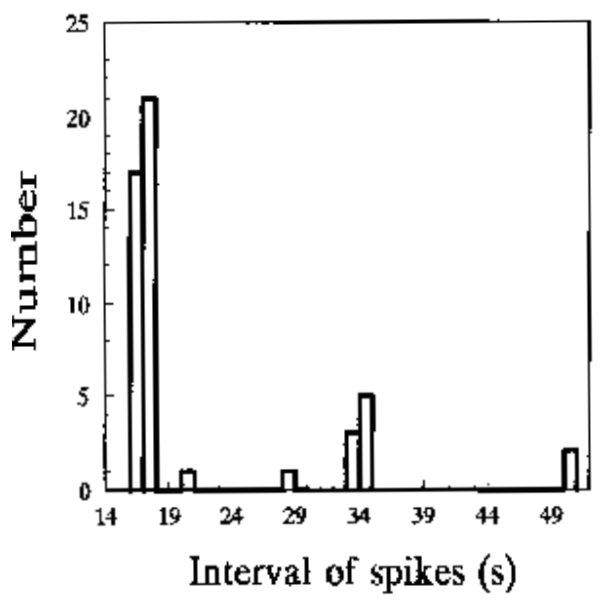

Fig. 5. The histogram for the intervals between the neighboring spikes.

should not exceed $10^{23}$ ergs, which still is orders of magnitude smaller than flares or microflares energy contents.

\subsection{Estimation of magnetic field of spike sources}

A simple and direct estimate of coronal magnetic field strength is provided by the gyroresonant emission at microwaves. Such emission occurs at discrete harmonics of the gyrofrequency, $f_{\mathrm{b}}=2.8 B_{\text {Gauss }} \mathrm{MHz}$ (White et al. 1991). We thus have $f=$ $s f_{\mathrm{b}}=2.8 \mathrm{~s} B_{\text {Gauss }} \mathrm{MHz}$, where $f$ is the frequency of the gyroresonant emission, $f_{\mathrm{b}}$ the electron gyrofrequency, $\mathrm{s}$ the harmonic number $(1,2,3 \ldots)$, and $B_{\text {Gauss }}$ the magnetic field strength in Gauss. Assuming that the emission of spikes can be attributed to the electron-cyclotron maser (ECM) instability, the $x$-mode at the second harmonic would be predominant in the growth rate of ECM instability (Winglee \& Dulk 1986). When the frequency of the gyroresonant emission $f$ equals the observing frequency $(1.42 \mathrm{GHz})$, we obtain an approximate magnetic field strength of 250 Gauss.

\subsection{Time structures of the spikes}

\section{1) The Quasi-periodicity}

53 FFSs (called microflares) were recorded during the whole process of the radio burst at $1.42 \mathrm{GHz}$ on 


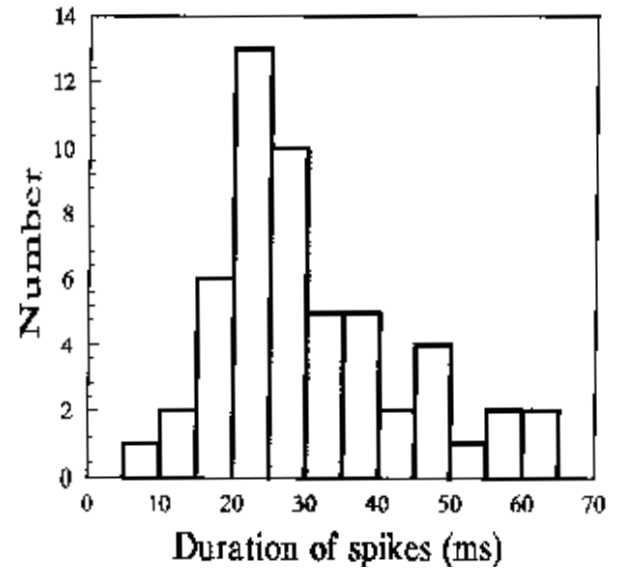

Fig. 6. The histogram for the durations of the spikes.

January 5, 1994, and all are isolated spikes forming a train with a long period. Typical samples of FFSs are shown in Fig. 1 (right columns). The time intervals $(\Delta t)$ between spikes are longer than those mentioned by Benz (1986). We did a statistical study of $\Delta t$ values and the results are shown in Fig. 5. We found that the $\Delta t$ between spikes were of $17 \mathrm{~s}$ for 39 events (76\% of the total spike number), $34 \mathrm{~s}$ for 9 events (16\%), and 21, 29, 50 and $50 \mathrm{~s}$ for the remaining 5 events. Figure 5 shows two obvious quasi-periods of $17 \mathrm{~s}$ and $34 \mathrm{~s}$ for the time interval between spikes. The quasi-periodicity may be due to the coalescence of two magnetic islands (see Tajima et al. 1987), or the explosive processes acting during magnetic reconnection (Tajima \& Sakai 1986; Litvinenko 1996).

\section{2) Similar shapes and durations}

Most of the spikes studied in this paper appear to have a similar shape: they rapidly rise and fall, presenting a symmetrical pattern. The distribution of spike durations shown in Fig. 6 reveals that it is in the range $15-50 \mathrm{~ms}$ for most of the fast structures, $55 \%$ of them with durations of about $20-30 \mathrm{~ms}$. Consequently, there is not a large dispersion in the observed energy and duration distributions.

The observed spikes may have been produced by a discontinuous exciter (e.g., Benz 1985), resulting in almost quantized small energy releases, as proposed by Güdel et al. (1991). This also could explained the relatively low dispersion in the distributions of the observed spike durations and radio energy contents.

\section{3) Double-peak structures}

It is notable that 18 out of the 53 observed spikes appear as discrete double-peak structures. This particular fact can be accounted for by the current loop coalescence model. Indeed, calculations and simulations (Tajima et al. 1987) have shown that during such scenario quasi-periodic oscillations of particle quantities, as well as double-peak structures in the electric field energy may develop.

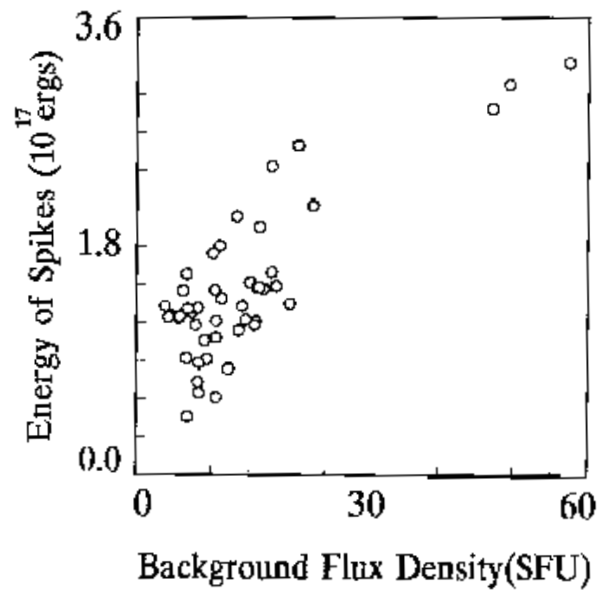

Fig. 7. The correlogram of the radio spike energy with the continuum background flux density.

\section{4) Correlation between the radio spike energy and the continuum background flux density}

Except for several isolated spikes, most of the observed fast time structures have been observed superimposed on a gradual background. Figure 7 indicates a suggestion for a linear relation between the spike radio energy content and the underlying radio flux density.

The observed millisecond spikes are indicators of nonthermal energy releases due to the acceleration of electrons. On the other hand the underlying gradual component could be due to thermal gyroresonance emission (Gopalswamy et al. 1997), as supported by the presence of simultaneous small gradual radio bursts in the centimeter and short decimeter wavelength ranges (S.G.D. 1994, 594 - part I). The linear relation between the radio energy content for the two components could also indicate that the gradual one is formed by the superposition of many weak, short duration, and unresolved spikes or group of spikes (Gary et al. 1991).

\section{Concluding remarks}

\section{1) The spikes as microflares}

The microflares are substantially small energetic transient phenomena. The transient energy releases involving $10^{26} \mathrm{ergs}$ down to a few times $10^{24}$ ergs have been detected using radio techniques (Bastian et al. 1998). For the present observations, the radio energy content of a spike might be corresponding to $\leq 10^{23}$ ergs released (see Sect. 3.1). This is only a small fraction of the total energy $\left(10^{26} \mathrm{ergs}\right)$ released in the largest hard X-ray microflares (Lin et al. 1984), and comparable to $\sim 10^{24}$ ergs of the thermal energy content of the TMBs (Gopalswamy et al. 1997). Therefore, we consider that each observed millisecond spike may reflect the behaviour of a microflare. The earlier interpretation of a single spike as a microflare suggested that spikes emission may be instantaneous radiation of a microflare, or thousands of them (Benz 1985 and 1986). In recent years, Fleishman \& Mel'nikov (1998) point out other possible interpretations for spikes: i) the intrinsic properties of the radio 
emission mechanism generation; ii) a "secondary" fragmentation of the radio emission source structure. The latter may be supported by our observations. As for the nonthermal electron acceleration site, it may be come from the separatrix region (about $1.3 \mathrm{GHz}$ ) of the pair type III bursts (Fig. 2).

It has been known that the spatial characteristics of radio spikes deal with both the microscopic emission mechanism and the plasma emission region itself. The separate spike sources (with characteristic size $\ell<200 \mathrm{~km}$ and $\Delta B / B \leq \Delta f / f \sim$ $0.01-0.08$ ) can distribute (randomly in time and space) inside a more extended plasma region, resulting in a spike cluster source. Each local source would generate a single spike at the moment (Fleishman \& Mel'nikov 1998). Also, during the development of a solar burst electron acceleration occur in different very localized sites distributed over a large area of the active region (Raulin et al. 2000). Therefore, only a small part of the spikes can be observed at a single frequency.

\section{2) The emission process of spikes}

We may interpret the observed microflares in terms of the quasi-linear plasma emission mechanism (Fleishman \& Mel'nikov 1998) and the current loop coalescence model (Krüger et al. 1994). In the quasi-linear regime a pulse of plasma waves is transformed into electromagnetic waves generating a radio pulse, which can produce a superfine millisecond time structure on time scales of several tens of milliseconds with periodic oscillation. The coalescence is proceeded by a sequence of elementary coalescence events with timescale down to $\sim 20 \mathrm{~ms}$, approaching the durations of the observed FFSs.

\section{Summary and conclusions}

Our results have confirmed the presence of millisecond spike emission during a radio burst at $1.42 \mathrm{GHz}$. The magnetic field strength B has been estimated to be $\sim 250$ Gauss, which is characteristic of a coronal field. The energy radiated during spikes is in the range $(0.3-3.3) \times 10^{17} \mathrm{ergs}$, which could correspond to $\leq 10^{23}$ ergs actually transferred to nonthermal electrons. In that sense, the observed spikes can be considered as radio microflares. The main properties of the spikes as microflares are summarized as follows: a spike train consists of 53 FFSs, with two quasi-periods ( $\sim 17$ and $34 \mathrm{~s}$ ). The average energy and duration of the spikes are about $1.4 \times 10^{17} \mathrm{ergs}$ and $33.1 \mathrm{~ms}$, respectively. The spikes are composed of single and double-peak structures with similar durations and morphologies.

The millisecond spikes as radio microflares may be similar to the hard X-ray microflares or TMBs, and other manifestations of small transient energy releases. They could reflect small-scale heating and/or particle acceleration in compact magnetic flux loops, arising from regions of bipolar or tripolar magnetic structures.

Acknowledgements. The authors are indebted to Drs. D. Luan and W. B. Li of the Yunnan Observatory for providing optical data and helpful discussions. This research is supported by the National NSF of China grant (No. 19833050 and 19973016), and the "973" project (No. G2000078403) and the foundation of the Chinese Academy of Sciences. We are grateful to the referee for helpful suggestions and encouragement.

\section{References}

Aschwanden, M. J., Benz, A. O., Schwartz, R. A., et al. 1990, Sol. Phys., 130, 39

Aschwanden, M. J., \& Güdel, M. 1992, ApJ, 401, 736

Aurass, H., Chernov, G. P., Karlický, M., et al. 1987, Sol. Phys., 112, 347

Benz, A. O. 1985, Sol. Phys., 96, 357

Benz, A. O. 1986, Sol. Phys., 104, 99

Bastian, T. S., Benz, A. O., \& Gary, D. E. 1998, ARA\&A, 36, 131

Csillaghy, A., \& Benz, A. O. 1993, A\&A, 274, 487

Fleishman, G. D., \& Mel'nikov, V. F. 1998, Physics-Uspekhi, 41(12), 1157

Fu, Q. J., Ji, H. R., Lao, D. B., et al. 1997, Acta Astrophys. Sinica, 17, 441

Gary, D. E., Hurford, G. J., \& Flees, D. J. 1991, ApJ, 369, 255

Gopalswamy, N., Payne, T. E. W., Schmahl, E. J., et al. 1994, ApJ, 437,522

Gopalswamy, N., Zhang, J., Kundu, M. R., et al. 1997, ApJ, 401, L115

Güdel, M., Aschwanden, M. J., \& Benz, A. O. 1991, A\&A, 251, 285

Hanaoka, Y. 1997, Sol. Phys., 173, 319

Hudson, H. S. 1991, Sol. Phys., 133, 357

Ji, H. R., Fu, Q. J., \& Lao, D. B. 1997, Acta Astron. Sin., 17, 219

Karlický, M., Aurass, H., \& Mann, G. 1986, Sol. Phys., 104, 165

Kuijpers, J. 1980, IAU Symp., 86, 341

Lin, R. P., Schwartz, R. A., Kane, S. R., et al. 1984, ApJ, 283, 421

Litvinenko, Yu. E. 1996, Sol. Phys., 167, 321

Porter, J. G., Moore, R. L., Reichmann, E. J., et al. 1987, ApJ, 323, 380

Raulin, J.-P., Vilmer, N., Trottet, G., et al. 2000, A\&A, 355, 355

Stähli, M., \& Benz, A. O. 1987, A\&A, 175, 271

Tajima, T., Sakai, J., Nakajima, H., et al. 1987, ApJ, 321, 1031

Tajima, T., \& Sakai, J. I. 1986, IEEE Trans, Plasma Sci., Ps-14, 929

Trottet, G. 1986, Sol. Phys., 104, 145

Ulmschneider, P., Rosner, R., Priest, E. R. (eds.) 1991, Mechanisms of Chromospheric and Coronal Heating (Springer-Velag, Berlin)

White, S. M., Kundu, M. R., Shimizu, T., et al. 1995, ApJ, 450, 435

Wild, J. P., Smerd, S. F., \& Weiss, A. A. 1963, ARA\&A, 1, 291

Winglee, R. M., \& Dulk, G. A. 1986, Sol. Phys., 104, 93

Xie, R. X., Yang, K. P., Li, W. H., et al. 1989, Acta Astron. Sin., 9(1), 77 